\title{
Study on the Relativity between Cytogenetics and Cytomorphology and its Prognosis Significance in Children with Acute Myelogenous Leukemia
}

\author{
Mehta $\mathrm{S}^{1^{*}}$, Singh $\mathrm{S}^{2}$, Wang Ning Ling ${ }^{3}$ \\ ${ }^{1}$ Consultant Pediatrician, Department of Pediatrics, Koshi Zonal Hospital, Biratnagar, Nepal. \\ 2 Lecturer, Coordinator, BSc.MIT Program, Gandaki Medical College \& Teaching Hospital, Pokhara, Nepal. \\ ${ }^{3}$ Department of Pediatrics, Second Affiliated Hospital of Anhui Medical University, Hefei, China.
}

\section{Keywords}

Abnormal karyotypes,

Acute myeloid leukemia,

Cytogenetics, Prognosis..

\section{Corresponding author}

*Dr. Subash Mehta

Consultant Pediatrician

Koshi Zonal Hospital

Morang, Koshi, Nepal

Email:drmehtasubash@gmail.com

\begin{abstract}
Objective: The main objective of this study was to retrospectively evaluate that the cytogenetic abnormalities is an important prognostic factor for the cure of acute myeloid leukemia (AML).

Methods: This retrospective study enrolled newly diagnosed 70 cases (37 males and 33 females, aged 10.1 months to 14.5 years) of pediatric patients with AML during 2010 January - 2016 February from the Second Affiliated Hospital of Anhui Medical University. Excluding criteria were cases secondary to treatment-related MDS and AML. Samples were obtained from bone marrow cells in patients after treatment on the anterior superior iliac spine, blood diseases laboratory by direct culture or 24/48 hour short-term culture, G -banding technique for testing. Follow-up of 1 - 60 months, the analysis of treatment response rates of different karyotypes, distribution ratios in various subtypes, normal karyotype and abnormal karyotype.
\end{abstract}

ISPSS17.0 software statistics was used for statistical analysis. Groups were compared using chi-square test; Survival rate was calculated by method of Kaplan Meier and survival difference between groups were compared with breslow test.

Results: Among 70 cases, 42 cases were detected for chromosomal abnormalities (i.e. $60 \%$ of the total number of cases), M3 abnormal karyotype distortion rate of 78.5\%, M2 abnormal karyotype aberrations 63.3\%, M4 60.0\%, M1 50\%, M5 lowest 38.9 \%, M7 nuclear aberrations highest rate was $100 \%$. Total chromosomal aberration rate was $60 \%$. Acute myeloid leukemia cases, $t(8 ; 21)$ at most, there are 15 cases, and the presence of abnormal karyotype $86.7 \%$ in the original part of differentiated myeloid leukemia (M2); t $(15 ; 17)$ has 11 cases, exists only in acute promyelocytic cell leukemia (M3). After treatment, the remission rate of $t(8 ; 21)$ was $80 \%$; the remission rate of $t(15 ; 17)$ was $90 \%$; the remission rate of other abnormal karyotype abnormalities was $50 \%$; the remission rate of total abnormal karyotype was $71.4 \%$. The event free survival rate was significantly different between normal karyotype, t (8; $21), t(15 ; 17)$ and other abnormal karyotype groups $(\mathrm{P}<0.05)$.

Conclusions: Acute myeloid leukemia karyotype abnormalities among FAB subtypes are different; M3 is the highest rate of abnormal 
karyotype aberrations, M2, M4 medium, M5 minimum. t (15; 17) seen in acute promyelocytic leukemia (APL), prognosis is good; $t(8 ; 21)$ is more common in M2, prognosis is good, also found in M4 and M5, worse prognosis; +8 Abnormalities found in AML M2, M3, M4, M5 and M6 subtypes, prognosis medium; inv (16) high white blood cells, low platelet poor prognosis, AML patients with normal karyotype prognosis medium.

\section{NTRODUCTION}

Acute Leukemia belong to the group of hematopoietic stem cell mutation clone malignancy which is characterized by certain blood cells of hematopoietic tissue system hyperplasia, and infiltration to the bloodstream and then to various tissues and organs, which led to a series of clinical manifestations.

In China, pediatric malignancies have the highest incidence of leukemia. With the continuous improvement of chemotherapy, the rapid development of immunology, cytogenetic and molecular genetics, it is no longer considered a fatal disease. Complete remission rate of initial treatment in children with acute myeloid leukemia has reached $80 \%$ and five year disease-free survival rate of about $40-60 \%$.

Over the past years, blood disease research work caught committed to explore the pathogenesis of acute leukemia from cytogenetic and molecular level that the considerable number of leukemia is chromosomal changes because of genetic abnormalities. Oncogene activation, tumor suppressor gene deletion, causing the proliferation of hematopoietic cells, differentiation and regulation disorders, to malignant transformation.

With the advancement of cultivation method and banding techniques, currently age-related macular degeneration (AMD) chromosomal aberrations detected rate has risen from $50 \%$ in the 1960 s and 1970 s and the current to around $80 \%-90 \%$. Although the type of AML chromosomal aberrations up to 100 or more, But it can be summarized into two categories: one is FAB subtypespecific chromosomal rearrangements related, about $60 \%$, including the $\mathrm{t}(8 ; 21)(\mathrm{q} 22 ; \mathrm{q} 22), \mathrm{t}(15 ; 17)$ (q 22; q12 or 21), inv (16) (p13; q22) etc.

Where in $\mathrm{t}(8 ; 21)$ (q22; q22) is closely related to AML-M2, $t(15 ; 17)$ (q22; q12 or 21) found only in AML-M3, inv (16) (p13; q22) more common in M4EO. Such karyotype on early diagnosis and classification of AML has a guide. FAB subtype and the other is not related to abnormal, most of the number of abnormal, common chromosome number has increased first 8, 21, 19, 11 and 22 there appears chromosome loss, Y chromosome 7, 5 and 12, common chromosome 16 deletion of the short arm missing 6 and No. 7, 5 and 1, 12, 3. Among them +8, -7, -5 are the most.

Over the years, more cooperative groups abroad, including SWOG (South West Oncology Group), MRC (Medical Research Council) analyzed by a large sample of cases, confirmed cytogenetic findings prognosis and significance for acute myeloid leukemia. In accordance with the prognosis of low-risk group divided cytogenetic risk groups and high-risk groups that differences in each group complete remission rate, overall survival, disease free survival time were significant, although each between the study groups of individual cytogenetic abnormalities prognostic significance remains controversial, but most of the prognostic significance of cytogenetic abnormalities results are consistent and clear.

Simple $t(8 ; 21)$, and with $t(15 ; 17)$, the general prognosis in patients with inv (16) change is good, with $-7,-5$, inv (3) (q21; q26) / t (3; 3) (q21; q26) or complex karyotype chromosome abnormalities, poor prognosis. Thus, by cytogenetic characteristics of prognostic evaluation, selection the appropriate treatment is essential to prolong the survival rate of patients.

In this study, 70 cases of hospitalized patients with recently diagnosed AML from January 2009-2015 February were enrolled with karyotype abnormalities, patients with acute myeloid leukemia were involved in the treatment to understand AML patients karyotype distribution, survival follow-up and outcome combined with the clinical diagnosis and treatment. The aim is to analyze the correlation between karyotype and prognosis.

\section{METHODS}

\subsection{Study}

Newly diagnosed 70 cases (37 males and 33 females, aged 10.1 months to 14.5 years) of pediatric patients with AML were enrolled from 2009 January - 2016 February from the Second Affiliated Hospital of Anhui Medical University. 
Excluding criteria were cases secondary to treatment related MDS and AML.

By FAB classification, M1 subtype 2 cases, M2 subtype 30 cases, M3 subtype 14 cases, M4 subtype 5 cases, M5 subtype 18 cases, and M7 subtype, one case. Diagnosis and evaluation of the efficacy of all patients were done with reference to "blood disease diagnosis and treatment standards"1.

\subsection{Specimen collection}

Patients admitted to hospital after chromosome analysis, samples were obtained from the bone marrow cells before and after treatment in patients from iliac spine. Five $\mathrm{ml}$ injection containing two $\mathrm{ml}$ broth, culture bottles were immediately taken for inspection.

\subsection{Cytogenetic analysis}

The samples were submitted to the Second Affiliated Hospital of Anhui Medical University, Laboratory of Hematology line karyotype. Direct method or short-term legal culture piece, karyotype analysis using G banding analysis of 20 metaphase cells, press "Human cytogenetic international nomenclature system (ISCN1995)" were to be carried out ${ }^{2}$.

At least two cells having the same or increase in chromosome structural rearrangements, or three cells having the same chromosome loss, will be confirmed as an exception clone.

\subsection{Treatment and efficacy evaluation}

Patient with recently diagnosed M3 with all-trans retinoic acid (ATRA) $25 \mathrm{mg} /\left(\mathrm{m}^{2} \bullet \mathrm{d}\right)$ and arsenic trioxide $\left(\mathrm{As}_{2} \mathrm{O}_{3}\right)$ $0.2 \mathrm{mg} /(\mathrm{kg} \bullet \mathrm{d})$ during induction therapy period, 28 days of oral. In consolidate the treatment period, patient were treated with cytarabine (Ara-C) $100 \mathrm{mg} /\left(\mathrm{m}^{2} \bullet \mathrm{q} 12 \mathrm{~h}\right)$ from one to seven days and DNR $40 \mathrm{mg} /\left(\mathrm{m}^{2} \bullet \mathrm{d}\right)$ from one to three days, fowling with ATRA $25 \mathrm{mg} /\left(\mathrm{m}^{2} \bullet \mathrm{d}\right)$ and As203 $0.2 \mathrm{mg} /(\mathrm{kg} \bullet \mathrm{d})$ for 28 days, and the very next treatment with Ara-C $100 \mathrm{mg} /\left(\mathrm{m}^{2} \bullet \mathrm{q} 12 \mathrm{~h}\right)$ from one to seven days and DNR $40 \mathrm{mg} /\left(\mathrm{m}^{2} \bullet \mathrm{d}\right)$ from one to three days. After completing the consolidating the treatment, patient entered maintenance treatment with ATRA 25 $\mathrm{mg} /\left(\mathrm{m}^{2} \bullet \mathrm{d}\right)$ for 28 days, Ara-C $75 \mathrm{mg} /\left(\mathrm{m}^{2} \bullet \mathrm{q} 12 \mathrm{~h}\right)$ for five days, harringtonine $3 \mathrm{mg} /\left(\mathrm{m}^{2} \bullet \mathrm{d}\right)$ for seven days, As203 $0.2 \mathrm{mg} /(\mathrm{kg} \bullet \mathrm{d})$ for 14 days, Ara-C $75 \mathrm{mg} /\left(\mathrm{m}^{2} \bullet \mathrm{q} 12 \mathrm{~h}\right)$ and 6-TG $75 \mathrm{mg} /\left(\mathrm{m}^{2} \bullet \mathrm{d}\right)$ for 7 days; five times totally. Efficacy criteria: Zhang Zhinan reference "blood disease diagnosis and treatment standards" ${ }^{11}$. Complete remission (CR); it means that the leukemic cells without clinical presentation caused by infiltration, or near normal life values $=1.5 \times$ $10^{9} / \mathrm{L}$, platelets $=100 \times 10^{9} / \mathrm{L}$. Peripheral blood leukocyte no leukemia cells. Bone marrow blasts $=5 \%$, red blood cells and megakaryocytes normal series. Partial remission (PR): Bone marrow myeloblast type I + type II (original ten immature monocytes or ten original immature lymphocytes) equals to $>5 \%$ and $=20 \%$, or clinically, a standard blood counts by the end of complete remission No remission (NR): None of the above criteria. Recurrence was defined by one of the following three criteria including (1) bone marrow blasts $>5 \%$ and $=20 \%$, after a course of an effective anti-leukemic treatment, bone marrow failed to achieve complete remission. (2) Bone marrow blasts $>20 \%$; (3) myeloid leukemia cell infiltration. Overall survival is defined as the time of diagnosis until death or end of follow-up. Disease-free survival is defined as the time to achieve complete remission to relapse during this time, if more than one relapse and remission as per the cumulative basis.

\subsection{Experimental Method}

\subsubsection{Reagent}

(1) Medium: containing 20\% fetal calf serum and $20 \mu / \mathrm{ml}$ heparin RPMI 1640.

(2) Mitosis blockers: colchicines, formulated $5 \mu \mathrm{g} / \mathrm{ml}$ were used.

(3) Hypotonic: $0.075 \mathrm{~mol} / \mathrm{L}$ potassium chloride solution.

(4) Fixative: 1: 3 glacial acetic acid and methanol.

(5) Banding solution: $0.1 \%$ trypsin, $0.02 \%$ EDTA solution, pH 6.8 phosphate buffer solution, Tris solution 3\%.

(6) Giemsa staining solution: phosphate buffer solution concentration of $10 \%$, pH $6.8 \sim 7.4$.

\subsubsection{Step}

(1) Take specimens and inoculation culture: Sterile needle to extract bone marrow fluid $0.5 \sim 2 \mathrm{~mL}$ in heparin tube, add broth to $8 \mathrm{~mL}$, washing and percussion, centrifugation (1200 rpm/min, $5 \mathrm{~min}$ ), repeated 2 - 3 times to wash away the fat and other impurities. Per $\mathrm{ml}$ medium $1 \sim 3 \times 106$ cells were inoculated to a sterile culture medium containing $5 \mathrm{ml}$ in to two flasks, shake gently and placed in the box 37 for 48 hours.

(2) Colchicines treatment: Sample bottle was added with colchicines $50 \mu \mathrm{l}$, using liquid $0.05 \mathrm{ml}$ (final concentration $0.05 \mu \mathrm{g} / \mathrm{mL}$ ) to continue to foster $50 \mathrm{~min}$. 


\section{(3) Preparation of samples:}

1) After termination of the culture, the culture was poured into the conical centrifuge tube, 6 minutes centrifugation (1200 rev/ min), the supernatant was discarded.

2) The prewarmed $8 \mathrm{ml}$ hypotonic solutions added by repeatedly pipetting at $37^{\circ} \mathrm{C}$ with bubble method, water bath for 30 minutes.

3) PreFixed: Add fixative $1 \sim 5 \mathrm{~mL}$, percussion mix placed under room temperature $3 \sim 5 \mathrm{~min}$.

4) Centrifugation: 800 to $1000 \mathrm{rev} / \mathrm{min}$.

5) Fixed: The supernatant was discarded, freshly prepared fixative was added to $5 \sim 6 \mathrm{~mL}$, mix by pipetting.

6) Centrifugation: 800 to $1000 \mathrm{rev} / \mathrm{min}, 10$ minutes

7) Repeat 5 and 6 steps two times.

8) The supernatant was discarded, adding an appropriate amount of fixative gently pipetting the cell suspension to spare.

9) Drop sheet: Take number of slides that stored in clean ice cold wet sheets, slightly tilted, with a capillary dropper suction cell suspension, dropping from 10 $\mathrm{cm}$ height of one to two drops on the slide in the air to be dry.

10) Roasted pieces: slides placed in $70^{\circ} \mathrm{C}$ oven bake for two hours.

\section{(4) G-banding:}

1) The first, vat $25 \mathrm{~mL}(0.1 \%)$ mixed with trypsin 25 $\mathrm{mL}(0.02 \%)$ EDTA solution, Tris was adjusted to $\mathrm{pH}$ 7. Second, three vat $50 \mathrm{~mL}$ were added at $\mathrm{pH} 6.8$ phosphate buffer. The fourth was added $50 \mathrm{~mL}(5 \%)$ Giemsa dye vat. The three dye vats were kept at $37^{\circ} \mathrm{C}$ water bath.

2) Slide into the first cylinder 20 - 30 seconds, remove successively rinsed in the second, three-cylinder, followed by the third set dye vat five to 10 minutes.

3) Tap water, dry equipment seized.

4) Each patient observed for at least 20 metaphase cells, abnormal karyotype press "Human Cytogenetic Nomenclature System (ISCN 1995)" are identified and described.

\subsection{Statistical Methods}

ISPSS17.0 software statistics was used for statistical analysis. Groups were compared using chi-square test;
Survival rate was calculated by method of Kaplan Meier and survival difference between groups were compared with breslow test.

\section{RESULTS}

Among 70 cases, 42 cases were detected for chromosomal abnormalities, i.e. $60 \%$ of the total number of cases.

Table 1: Karyotype distribution

\begin{tabular}{lcccc}
\hline $\begin{array}{c}\text { FAB sub- } \\
\text { type }\end{array}$ & $\begin{array}{c}\text { Number } \\
\text { of cases }\end{array}$ & $\begin{array}{c}\text { Normal } \\
\text { karyotype }\end{array}$ & $\begin{array}{c}\text { Abnormal } \\
\text { karyotype }\end{array}$ & $\begin{array}{c}\text { Distortion } \\
\text { rate (\%) }\end{array}$ \\
\hline M1 & 2 & 1 & 1 & $50 \%$ \\
M2 & 30 & 11 & 19 & $63.3 \%$ \\
M3 & 14 & 3 & 11 & $78.5 \%$ \\
M4 & 5 & 2 & 3 & $60.0 \%$ \\
M5 & 18 & 11 & 7 & $38.9 \%$ \\
M7 & 1 & 0 & 1 & $100 \%$ \\
Total & $\mathbf{7 0}$ & $\mathbf{2 8}$ & $\mathbf{4 2}$ & $\mathbf{6 0 \%}$ \\
\hline
\end{tabular}

As can be seen from Table 1, all 70 patients with acute myeloid leukemia, M1 subtype one case of abnormal karyotype; M2 subtype 19 cases of abnormal karyotypes, M3 subtype 11 cases of abnormal karyotypes; M4 subtype three cases of abnormal karyotypes, M5 subtype seven cases of abnormal karyotypes; M7 subtypes one case of abnormal karyotype. M3 abnormal karyotype distortion rate of $78.5 \%$, M2 abnormal karyotype aberrations 63.3\%, M4 60.0\%, M1 50\%, M5 lowest, 38.9\%, M7 nuclear aberrations highest rate was $100 \%$. However, due to a number of cases investigated test is too small, it is no clear statistical significance between this groups. So this experiment shows M3 subtype highest distortion, total chromosomal aberration rate $60 \%$.

Table 2: Abnormal karyotype in AML

\begin{tabular}{|c|c|c|c|c|c|c|}
\hline \multirow{2}{*}{$\begin{array}{c}\text { Abnormal karyo- } \\
\text { type }\end{array}$} & \multicolumn{5}{|c|}{ FAB Subtype } & \multirow{2}{*}{ Total } \\
\hline & M1 & M2 & M3 & M4 & $\begin{array}{ll}\text { M5 } & \text { M7 }\end{array}$ & \\
\hline$t(8 ; 21)$ & & 13 & & & 2 & 15 \\
\hline$t(5 ; 17)$ & & & 11 & & & 11 \\
\hline Inv (16) (13; q22) & & & & 1 & 1 & 2 \\
\hline $8 q-$ & & 2 & & & & 2 \\
\hline $11 p+$ & & 1 & & & & 1 \\
\hline $11 q^{-}$ & & 1 & & & & 1 \\
\hline+8 & & & & 1 & & 1 \\
\hline+12 & & & & 1 & & 1 \\
\hline-7 & 1 & & & & & 1 \\
\hline-19 & & 1 & & & & 1 \\
\hline
\end{tabular}




\begin{tabular}{ccccccccc}
$\begin{array}{c}\text { Hyperdiploidy } \\
\begin{array}{c}\text { Complex abnormal- } \\
\text { ities }\end{array}\end{array}$ & 1 & & & 3 & & 4 \\
Total & $\mathbf{1}$ & $\mathbf{1 9}$ & $\mathbf{1 1}$ & $\mathbf{3}$ & $\mathbf{7}$ & $\mathbf{1}$ & $\mathbf{4 2}$ \\
\hline
\end{tabular}

Table 2 shows that, in acute myeloid leukemia patients, there are 10 kinds of abnormal karyotype and complex chromosomal aberrations. $t(8 ; 21)$ at most, 15 cases, and the presence of abnormal karyotypes was $86.7 \%$ in the original part of differentiated myeloid leukemia (M2); t $(5$; 17) 11 cases, exists only in acute promyelocytic cell leukemia (M3) , inv (16) (p13; q22) in two cases, respectively, in the presence of the M4 and M5, 8q- two cases exist in M2, $11 \mathrm{p}+1$ case and $11 \mathrm{q}-$, are present in $\mathrm{M} 2$, the +8 and +12 in one case, all exist in M2, the -19 one case, exist in $\mathrm{M} 2$, four patients hyperdiploidy were present in M2 and M5, and the remaining two cases were complicated karyotype, they were present in the M5 and M7.

Table 3: Abnormal karyotype and efficacy in AML

\begin{tabular}{lcccc}
\multicolumn{1}{c}{ Group } & CR+PR & NR & Total & $\begin{array}{c}\text { Response } \\
\text { Rate (\%) }\end{array}$ \\
\hline $\mathrm{t} \mathrm{(8;} \mathrm{21)}$ & 12 & 3 & 15 & 80 \\
$\mathrm{t}(5 ; 17)$ & 10 & 1 & 11 & 90.1 \\
$\begin{array}{l}\text { Other abnormal } \\
\text { karyotype }\end{array}$ & 8 & 8 & 16 & 50 \\
Total & $\mathbf{3 0}$ & $\mathbf{1 2}$ & $\mathbf{4 2}$ & $\mathbf{7 1 . 4}$ \\
\hline
\end{tabular}

Fig 1: The event free survival curves of different karyotype in AML

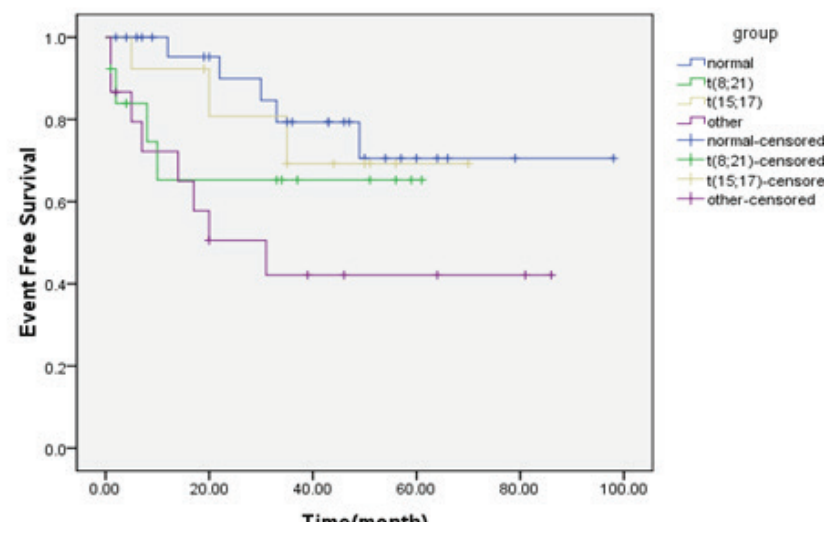

From table 2, table 3 and fig 1 can be seen, M2 subtype in 13 patients with abnormal karyotype $t$ (8; 21), of which 12 cases of remission after treatment, two cases are not alleviated after treatment, remission rate $80 \%$. M3 subtype in 11 patients with abnormal karyotype $t$
(15; 17), 10 patients were in complete remission after treatment, one case was not alleviated, and remission rate was $90.1 \%$. Other abnormal karyotypes total of 16 cases, $50 \%$ response rate. There are two cases which inv (16) (p13; q22), one case remission, and one died, two cases of $8 \mathrm{q}$ - were relieved, one case of $11 p+$, follow-up death, one case of 11 - was remission. One case of +8 was died. One case of +12 was remission. One case of -7 patients was followed up in death. One case of -19 was remission. The event free survival rate was significantly different between normal karyotype, $t(8 ; 21), t(15 ; 17)$ and other abnormal karyotype groups $(\mathrm{P}=0.028<0.05)$. Further statistical analysis found that event free survival rate free survival rate between normal karyotype and other abnormal karyotype groups was statistically difference $(\mathrm{P}=0.004<0.05)$, no difference between the rest of the groups.

\section{DISCUSSION}

With the continuous improvement of chromosome banding techniques, especially the rapid development of high-resolution technology, such chromosomal abnormalities relationship between certain types of leukemia and has become more and more closely, but also identified a number of subtypes iconic chromosome abnormal characteristics. Meanwhile, with disease progression or improvement of chromosomal abnormality occurs constantly changing, these changes we adjust has a significant role in the treatment and prognosis of the disease. In most patients, the application of cytogenetic methods can be found in clear cell clonal chromosomal abnormalities. The same chromosomal abnormalities are also seen in acute leukemia (AL) of each subtype, which means not only the diagnosis, but also prognostic value, the clinical characteristics of patients; help us to predict the patient's condition, because the merits of any karyotype changes are not absolute. Chromosomal abnormalities are important prognostic factor, such as $t$ $(8 ; 21)$ and $t(15 ; 17)$ or inv $(16)$ of better prognosis, and $-5,-7, \mathrm{t}(9 ; 22)$, and complex chromosomal abnormalities have poor prognosis ${ }^{3-4}$.

The group of 70 children with acute myeloid leukemia, chromosome aberrations was 60\%, suggesting that cytogenetic abnormalities are an important factor in the occurrence of acute myeloid leukemia. Highest karyotype aberration M3, 78.5\%, M5 lowest, 38.9\%, between the groups, not statistically significant. Considering the small sample volume chromosome aberration rate difference 
between the AML subtypes may vary.

\subsection{Acute promyelocytic leukemia (APL) and t $(15 ; 17)$}

This group included 14 patients with 11 cases of abnormal karyotype APL, where, t $(15 ; 17) 11$ cases, accounting for $100 \%$. Alan k Burnett ${ }^{5}$ and other studies of 218 patients with APL, 187 cases of $t(15 ; 17)$, accounting for 85.8\%, there was no difference between the present studies. Kühnl A and Grimwade D 6 studied 1612 cases of AML patients, 932 cases had abnormal karyotype, t (15; 17) 198 cases, accounting for $21.2 \%$ response rate was $87 \%$. The patients with $\mathrm{t}(15 ; 17)$ of the total 70 cases of abnormal karyotype AML $15.6 \%$ response rate was $100 \%$, more consistent both with David Grimwade and other reports. 10 cases of $t(15 ; 17)$ have been applied, all-trans retinoic acid treatment, 10 cases of remission, remission rate was $100 \%$. M3 has t $(15 ; 17)$ translocation PML (promyelocytic leukemia gene) on chromosome 15 and 17, retinoic acid receptor gene (RAR $\alpha$ ) chromosome formation PML-RAR $\alpha$ fusion gene, which is M3 incidence and application of alltrans retinoic acid treatment effective molecular basis. $\mathrm{t}$ $(15 ; 17)$ prognosis is good, has its foundation in molecular biology ${ }^{6-7}$.

\subsection{Acute myeloid leukemia part differentiation type (M2) and $t(8 ; 21)$}

$t(8 ; 21)$ is the first to be identified as chromosomal abnormalities in AML, seen in AML-M2, especially in childhood AML is more common, a few also found in AML-M4. In adults, $t(8 ; 21)$ prognosis is good, but in children, the prognosis is poor. Foucar $\mathrm{K}^{8}$ reported 11 cases of $\mathrm{t}(8 ; 21)$ patients, nine cases of $\mathrm{M} 2$, two cases of M4, M4's two cases were five and 11 years old, after allogeneic hematopoietic stem cell transplantation to achieve complete remission, nine cases $M 2$, seven cases of complete remission. $t(8 ; 21)$ showed no difference between the proportion of this group of patients. The patients in all AML t $(8 ; 21)$ a total of 13 cases, 10 cases of remission, remission rate was $78.5 \%, 86.7 \%$ seen in M2. Prigogina EL et $a l^{9}$ reported 88 cases of acute myeloid leukemia (AML) patients, 27 cases of $t(8 ; 21), 20$ cases of cases of M1, 3 cases of M2, 4 cases of M4, t (8; 21) in patients with abnormal karyotype prognosis than the normal karyotypes or other cytogenetic abnormalities better. John C. Byrd ${ }^{10}$ analyzed 1213 cases of de novo AML patients, 81 cases of $t(8 ; 21)$, the prognosis is good, and the remission rate of $91 \%$, more in line with this group, and the combined second abnormal karyotype or complex karyotypes does not affect the prognosis. $t$ (8; 21) rarely seen in M5, in Molero MT only one case reported ${ }^{11}$. The patients had abnormal karyotype +8 one case, accounting for $3.1 \%$ of the total AML, as M4, did not ease. Li $\mathrm{X}^{12}$ reported 2308 Chinese children and adults with de novo acute myeloid leukemia; abnormal +8 (5.5\%) +8, the prognosis is moderate, consistent with the results of this group. Vaskova $\mathrm{J}^{13}$ reported of $10 \%$ and other reported of $12 \%$ and $8 \%$. Sandra R. Wolman et al ${ }^{14}$ reported 849 cases of AML patients, 108 cases of +8 (12.7\%), 43 cases $(5.1 \%)+8$ as the only exception, the exception has +8 poor prognosis.

\subsection{Clinical relationship of karyotypes inv (16) (p13; q22)}

The patients of inv (16) (p13; q22) there are two cases, one case exists in M4, achieve remission, one case of M5, did not achieve remission. The patients were males, aged four, WBC $56.2 \times 10^{9} / \mathrm{L}$, platelets $12 \times 10^{9} / \mathrm{L}$; there are many adverse prognostic factors age, low platelets, and its poor prognosis. Eghtedar A, et al ${ }^{15}$ summarized 110 cases of inv (16) patients, CR 93\%, high white blood cell $(>120 \times 109 / \mathrm{L})$ and low platelets $\left(<30 \times 10^{9} / \mathrm{L}\right)$ difficult to achieve CR. $65.3 \%$, the prognosis is moderate.

\subsection{Clinical relationship of normal karyotype}

This group of patients with normal karyotype had 28 patients, seven cases of NR, response rate was $75 \%$, means for survival time is $78.5 \pm 7.5$ month. Torstein Haferlach ${ }^{16}$ and other reports analyzed 453 cases of newly diagnosed AML patients. It showed normal karyotype and abnormal +8 , prognosis medium.

\section{CONCLUSIONS}

This article summarizes 70 cases of children with acute myeloid leukemia untreated cases, the application of short-term culture and/ or direct method to do G-banding karyotype analysis, all patients of acute myeloid leukemia were given standard regimen and observed cytogenetic karyotype relations and efficacy, the following conclusions occur:

(1) Acute myeloid leukemia cell has its genetic basis, diagnosis of chromosomal studies in acute myeloid leukemia are important in treatment and prognosis. 
(2) Acute myeloid leukemia karyotype abnormalities among FAB subtypes are different; M3 is the highest rate of abnormal karyotype aberrations, M2, M4 medium, M5 minimum.

(3) $\mathrm{t}(15 ; 17)$ seen in acute promyelocytic leukemia (APL), prognosis is good.

(4) $t(8 ; 21)$ is more common in M2, prognosis is good, also found in M4 and M5, worse prognosis;

(5) +8 Abnormalities found in AML M2, M3, M4, M5, M6 subtypes, prognosis medium;

(6) inv (16) high white blood cells, low platelet poor prognosis;

(7) AML patients with normal karyotype prognosis are better than other groups.

This study belongs to the single center study with small sample, only one case of some subtypes, so, we will join with other centers to expand the samples and further improve this study.

\section{REFERENCES}

1. Zhang Zhinan. Blood disease diagnosis and efficacy criteria [M] 3 edition. Beijing: Science and Technology Press. 2007: 103-116.

2. Killick S, Matutes E, Powles RL et al. Outcome of biphenotypic acute leukemia. Haematologica. 2008; 84: 699-706.

3. Döhner H, Weisdorf DJ, Bloomfield CD. Acute myeloid leukemia. N Engl J Med. 2015; 373(12): 1136-52.

4. de Lima MC, da Silva DB, Freund AP et al. Acute Myeloid Leukemia: Analysis of epidemiological profile and survival rate. J Pediatr (Rio J). 2016 Feb 3.

5. Burnett AK. Treatment of acute myeloid leukaemia. Lin Med (Lond). 2013; 13 Suppl 6: s58-61.

6. Kühnl A, Grimwade D. Molecular markers in acute myeloid leukaemia. Int J Hematol. 2012; 96(2): 15363.

7. Grimwade D, Ivey A, Huntly BJ. Molecular landscape of acute myeloid leukemia in younger adults and its clinical relevance. Blood. 2016; 127(1): 29-41.

8. Foucar K, Anastasi J. Acute myeloid leukemia with recurrent cytogenetic abnormalities. Am J Clin Pathol. 2015; 144(1): 6-18.

9. Prigogina EL, Fleischm an EW, Puchkova GP et al. Chromosomes in acute nonlymphocytic leukemia. Hum Genet. 2009; 73(2): 137-46.

10. John C. Byrd, Krzyszt of Mro 'zek, Richard K. Dodge et al. Pretreatment cytogenetic abnormalities are predictive of induction success, cumulative incidence of relapse, and overall survival in adult patients with de novo acute myeloid leukemia: Results from cancer and Leukemia Group B (CALGB 8461). blood. 2002; $100(13):$ 4325-4336.

11. Molero MT, Gom ez Casares MT, Vale ncia JM et al. Detection of a $\mathrm{t}(8 ; 21)$ (q22;q22) in a case of M5 acute monoblastic leukemia. Cancer Genet Cytogenet. 2003; 100(2): 176-8.

12. Li $\mathrm{X}, \mathrm{Xie} \mathrm{W}, \mathrm{Hu} \mathrm{Y}$ et al. Comprehensive profile of cytogenetics in 2308 Chinese children and adults with de novo acute myeloid leukemia. Blood Cells Mol Dis. 2012; 49(2): 107-13.

13. Vaskova J, Dubayova $\mathrm{K}$, Cakanova G et al. Incidence and prognostic value of known genetic aberrations in patients with acute myeloid leukemia-a two year study. Klin Onkol. 2015; 28(4): 278-83.

14. Sandra R. Wolman, Holly Gundacker, Frederick R. Appelbaum et al. Impact of trisomy $8(+8)$ on clinical presentation, treatment response, and survival in acute myeloid leukemia: A South West Oncology Group study. Blood. 2002; 100(1): 29-35.

15. Eghtedar A, Borthakur G, Ravandi F et al. Characteristics of translocation $(16 ; 16)$ (p13; q22) acute myeloid leukemia. Am J Hematol. 2012; 87(3): 317-8.

16. Torsten Haferlach, Claudia Schoch, Helmut Löffler et al. Morphologic dysplasia in de novo acute myeloid leukemia (AML) is related to unfavorable cytogenetics but has no independent prognostic relevance under the conditions of intensive induction therapy: Results of a multiparameter analysis from the German AML Cooperative Group Studies. Journal of Clinical Oncology. 2003; 21(2): 256-265. 